\title{
Preface: Evolution of refractory grains, volatiles, and organic molecules from the interstellar medium to the early solar system
}

\author{
Shogo TACHIBANA, ${ }^{1 *}$ HeNNER BUSEMANN $^{2}$ and Lydie BONAL $^{3}$ \\ ${ }^{1}$ Department of Natural History Sciences, Hokkaido University, N10W8, Kita-ku, Sapporo, Hokkaido 060-0810, Japan \\ ${ }^{2}$ Inst. für Geochemie und Petrologie, ETH Zürich, NW C 84, Clausiusstrasse 25, CH-8092 Zürich, Switzerland \\ ${ }^{3}$ Institut de Planétologie et d'Astrophysique de Grenoble, IPAG/PLANETO, Bâtiment D de Physique - BP 53, \\ 38041 Grenoble Cedex 9, France
}

Primitive extraterrestrial materials record the early evolution of the solar system that predated planet formation. They also preserve the interstellar heritage from prior to the Sun's birth in the form of presolar grains and potentially as isotopic signatures of light elements in organic matter and specific molecules. It is one of the key issues in cosmochemistry to link the birth and evolution of the solar system to the evolution of the interstellar medium (ISM) and the Galaxy. To address this issue, an interdisciplinary approach linking analysis of extraterrestrial materials including samples returned by spacecraft, laboratory experiments, astronomical observations, and theoretical studies is crucial.

This special issue arose from a session "Refractory Grains, Volatiles, and Organic Molecules Inherited from the Interstellar Medium" at the Goldschmidt Conference (Aug. 25-30, 2013, Florence Italy) and consists of six articles covering a wide array of topics and disciplines to explore various perspectives of cosmochemistry; astronomical observation, laboratory experiments, extraterrestrial organic chemistry, astrophysical modeling, in-situ isotopic analyses, chronology, and sample-return mission.

Dartois et al. (2014) summarize observational and laboratory studies on interstellar carbonaceous dust to explore the link between ISM carbonaceous dust and insoluble organic matter (IOM) found in chondrites and interplanetary dust particles (IDPs). Several forms of carbonaceous solids are observed in the ISM (polyaromatic hydrocarbons (PAHs), amorphous carbons, hydrogenated amorphous carbons (a-C:H), fullerenes, and ices that further turn into macromolecular organic residues after ice-sublimation), among which PAHs, a-C:H and/or macromolecular organic residues would be the main precursor of solar system carbonaceous materials. PAHs and $\mathrm{a}-\mathrm{C}: \mathrm{H}$ are more aromatic and contain less oxygen and nitrogen than solar-system IOM. Organic matter formed

\footnotetext{
*Corresponding author (e-mail: tachi@ep.sci.hokudai.ac.jp)

Copyright (c) 2014 by The Geochemical Society of Japan.
}

by photolysis and/or radiolysis of ice in laboratories do not match the entire infrared spectral features of IOMs either. These lines of evidences indicate that further evolution of organic materials may have occurred in the early stage of solar-system formation from ISM carbonaceous dust as a precursor.

The evolution of organic matter in the early solar system may also be recorded in soluble organic components extracted from primitive meteorites. Yamashita and Naraoka (2014) report two homologous series of alkylpyridines $\left(\mathrm{C}_{n} \mathrm{H}_{2 n-5} \mathrm{~N}\right.$ and $\left.\mathrm{C}_{n} \mathrm{H}_{2 n-7} \mathrm{~N}\right)$ identified in the methanol extract of the Murchison meteorite by liquid chromatography/high-resolution mass spectrometry. The organic components consisting of $\mathrm{C}, \mathrm{H}$, and $\mathrm{N}$ are likely to have escaped chemical oxidation during parent body alteration and may thus record the early stage of formation of meteoritic organic components. Laboratory simulation experiments involving $\mathrm{HCHO}, \mathrm{CH}_{3} \mathrm{CHO}$, and $\mathrm{NH}_{3}$ produced alkylpyridines, which provided a plausible synthetic pathway for meteoritic alkylpyridines via aldol condensation and Chichibabin-type reactions (alkylpyridine formation from aldehydes and $\mathrm{NH}_{3}$ ). Their findings indicate the possible importance of aldehyde activity under alkaline conditions in the presence of ammonia for the chemical evolution of soluble organic matter in carbonaceous chondrites.

Water is one of the dominant volatile components in the solar system, and played many essential roles in solar system chemistry through interactions with minerals and organic matter. Tornow et al. (2014) model the formation, destruction and re-formation of water molecules during the earliest stage of star formation (a quasistationary molecular cloud core and its collapse to form a protostar) focusing on the evolution of the $\mathrm{D} / \mathrm{H}$ ratio. Their model show that the $\mathrm{D} / \mathrm{H}$ ratios of water in the gas and dust phases change spatially and temporally and that their $\mathrm{D} / \mathrm{H}$ ratios are higher than the solar-system meteoritic water. The model thus infers that the relatively low $\mathrm{D} / \mathrm{H}$ ratio of solar-system meteoritic water may be related to the later evolutional stage of the protosolar disk. 
The inner solar system water might have had low $\mathrm{D} / \mathrm{H}$ ratios, but it has not yet been clear how many water sources were present in the early solar system and how such water inventories interacted with each other. Yurimoto et al. (2014) report the first $\mathrm{H}$ and $\mathrm{O}$ isotope measurements of asteroidal liquid water, found as fluid inclusions in halite crystals from two $\mathrm{H}$ chondrites (Monahans and Zag), with a secondary ion mass spectrometer equipped with a cryo-sample stage (CryoSIMS). Highly variable $\mathrm{H}$ and $\mathrm{O}$ isotopic compositions were found in the fluids inclusions; $-400<\delta \mathrm{D}<+1300 \%$ o and $-20<\Delta^{17} \mathrm{O}<+30 \%$. This indicates that asteroidal aqueous fluids had at least two sources (inner solarsystem chondritic water and outer solar-system cometary water) and that the fluids with various mixing degrees were in isotopic disequilibrium before trapped into halite on the parent body (bodies) of $\mathrm{H}$ chondrites. Yurimoto et al. (2014) concluded that such various degrees of waterrock interaction on planetesimals may be the fundamental evolution pathway of planetary water. We should note that this new and challenging technological approach has allowed it to analyze the isotopic compositions of asteroidal fluids for the first time.

The dating of early solar-system materials is crucial to investigate the evolution of the solar system, and shortlived radionuclides such as ${ }^{26} \mathrm{Al}$ and ${ }^{53} \mathrm{Mn}$ have been used as high-precision chronometers. Nagashima et al. (2014) investigate $\mathrm{Al}-\mathrm{Mg}$ isotope systematics in mineralogically pristine chondrules from unmetamorphosed CR2 (Renazzo-type) carbonaceous chondrites with secondary ion mass spectrometry. The CR chondrites are among the most primitive chondrites; they experienced various degrees of aqueous alteration, but largely avoided thermal metamorphism. Resolvable ${ }^{26} \mathrm{Mg}$ excesses, correlated with $\mathrm{Al} / \mathrm{Mg}$ ratios, were observed in six out of 14 chondrules, and their inferred initial ${ }^{26} \mathrm{Al} /{ }^{27} \mathrm{Al}$ ratios, $\left({ }^{26} \mathrm{Al} /{ }^{27} \mathrm{Al}\right)_{0}$, ranged from $\sim 1 \times 10^{-6}$ to $\sim 6 \times 10^{-6}$. Other eight chondrules showed no detectable ${ }^{26} \mathrm{Mg}$ excesses with an upper limit of $\left({ }^{26} \mathrm{Al} /{ }^{27} \mathrm{Al}\right)_{0} \sim(2-3) \times 10^{-6}$. These lower $\left({ }^{26} \mathrm{Al} /{ }^{27} \mathrm{Al}\right)_{0}$ ratios for $\mathrm{CR}$ chondrules than those in other primitive chondrites indicate that high-temperature chondrule formation processes occurred in the CR chondrite forming region $\sim 1-2 \mathrm{Myr}$ later than other chondrite forming regions.

Sample-return missions such as Apollo, Stardust, Genesis, and Hayabusa, have contributed significantly to the progress of cosmochemistry. Tachibana et al. (2014) outline the Hayabusa2 sample-return mission to the C-type near-Earth asteroid $1999 \mathrm{JU}_{3}$ (2014-2020), focusing on the cosmochemical importance of returned samples from the asteroid. C-type asteroids are expected to preserve the most pristine materials in the solar system, a mixture of minerals, ice, and organic matter that interact with each other, and record the cosmochemical evolution from the interstellar medium to the early solar system. Space mis- sions are the only way to obtain such pristine materials with geologic context and without terrestrial contamination. The samples from the surface of this asteroid will also provide a precious opportunity to study current surface geological activities and the orbital evolution of the asteroid. The Hayabusa 2 and NASA's OSIRIS-REx sample-return mission to the B-type asteroid Bennu (2016-2023) (Lauretta et al., 2014) will open a new era in cosmochemistry in the early 2020 's.

Finally we are grateful to all the authors for their contributions and all the reviewers for their constructive and meaningful reviews. We also thank Prof. Hisayoshi Yurimoto, the chef editor of Geochemical Journal, and the journal editorial office for this precious opportunity of the special issue on cosmochemistry and their kind assistance and patience throughout the editorial process.

\section{REFERENCES}

Dartois, E., Engrand, C., Brunetto, R., Duprat, J., Pino, T., Quirico, E., Remusat, L., Bardin, N., Briani, G., Mostefaoui, S., Morinaud, G., Crane, B., Szwec, N., Delauche, L., Jamme, F., Sand, C. and Dumas, P. (2014) Interstellar and interplanetary carbonaceous solids in the laboratory. Geochem. J. 48, this issue, 511-518.

Lauretta, D. S., Bartels, A. E., Barucci, M. A., Bierhaus, E. B., Binzel, R. P., Bottke, W. F., Campins, H., Chesley, S. R., Clark, B. C., Clark, B. E., Cloutis, E. A., Connolly, H. C., Crombie, M. K., Delbó, M., Dworkin, J. P., Emery, J. P., Glavin, D. P., Hamilton, V. E., Hergenrother, C. W., Johnson, C. L., Keller, L. P., Michel, P., Nolan, M. C., Sandford, S. A., Scheeres, D. J., Simon, A. A., Sutter, B. M., Vokrouhlick, D. and Walsh, K. J. (2014) The OSIRIS-REx target asteroid 101955 Bennu: Constraints on its physical, geological, and dynamical nature from astronomical observations. Meteorit. Planet. Sci., 1-16, doi:10.1111/maps.12353.

Nagashima, K., Krot, A. N. and Huss, G. R. (2014) ${ }^{26} \mathrm{Al}$ in chondrules from CR2 chondrites. Geochem. J. 48, this issue, 561-570.

Tachibana, S., Abe, M., Arakawa, M., Fujimoto, M., Iijima, Y., Ishiguro, M., Kitazato, K., Kobayashi, N., Namiki, N., Okada, T., Okazaki, R., Sawada, H., Sugita, S., Takano, Y., Tanaka, S., Watanabe, S., Yoshikawa, M., Kuninaka, H. and the Hayabusa2 Project Team (2014) Hayabusa2: Scientific importance of samples returned from C-type near-Earth asteroid (162173) $1999 \mathrm{JU}_{3}$. Geochem. J. 48, this issue, 571587.

Tornow, C., Gast, P., Pelivan, I., Kupper, S., Kührt, E. and Motschmann, U. (2014) Simulating the D/H ratio of water formed in the early solar nebula. Geochem. J. 48, this issue, 527-547.

Yamashita, Y. and Naraoka, H. (2014) Two homologous series of alkylpyridines in the Murchison meteorite. Geochem. J. 48, this issue, 519-525.

Yurimoto, H., Itoh, S., Zolensky, M., Kusakabe, M., Karen, A. and Bodnar, R. (2014) Isotopic compositions of asteroidal liquid water trapped in fluid inclusions of chondrites. Geochem. J. 48, this issue, 549-560. 